\title{
Optimizing our patient's entropy production as therapy? Hypotheses originating from the physics of physiology
}

Andrew Ervine Seely ( $\square$ aseely@ottawahospital.on.ca )

\section{Research}

Keywords: Maximum Entropy Production Principle, Fractal Structures, Complex Nonequilibrium Systems, Monitoring of Scale-invariant Variation, Thermodynamics

Posted Date: May 28th, 2020

DOl: https://doi.org/10.21203/rs.3.rs-31100/v1

License: (c) (i) This work is licensed under a Creative Commons Attribution 4.0 International License. Read Full License 


\title{
Optimizing our patient's entropy production as therapy?
}

\section{Hypotheses originating from the physics of physiology}

\author{
Andrew J.E. Seely $1,2,3^{*}$ \\ ${ }^{1}$ Professor, University of Ottawa, Ottawa, Ontario, Canada \\ ${ }^{2}$ Scientist, Ottawa Hospital Research Institute, University of Ottawa, Ontario, Canada \\ ${ }^{3}$ Thoracic Surgery and Critical Care Medicine, University of Ottawa, Ottawa, Ontario, Canada
}

"Corresponding Author: Andrew JE Seely MD PhD FRCSC Ottawa Hospital - General Campus

501 Smyth Road, Box 708

Ottawa, Ontario, Canada, K1H 8L6

Phone: (613) 737-8899 ext. 74052

Fax: (613) 737-8668

E-mail: aseely@ohri.ca (publishable) 


\begin{abstract}
Physical laws dictate that energy is preserved; yet energy gradients irreversibly dissipate, thus producing entropy. As living complex non-equilibrium systems, humans must produce entropy continuously over time to create healthy internal emergent order. Entropy production is measured by heat production divided by temperature. Several hypotheses are presented. First, human entropy production is due to both metabolism and consciousness, dissipating energy and information gradients. Second, the physical drive for maximal entropy production is responsible for spontaneous formation of fractal multi-scale self-similar structures in time and space, ubiquitous and essential for health. Third, the evolutionary drive for enhanced function and adaptability selects states with both robust basal and maximal entropy production (i.e. the capacity to augment it when required). Last, targeted focus on optimizing our patients' entropy production will improve health and clinical outcomes. These hypotheses have implications for understanding health, metabolism and consciousness, and offer novel clinical treatment strategies.
\end{abstract}

Keywords: Maximum Entropy Production Principle; Fractal Structures; Complex Nonequilibrium Systems; Monitoring of Scale-invariant Variation, Thermodynamics 


\section{Introduction}

Physicians are naturally physicists at heart, seeking to understand why their patients are ill, so as to improve their care. Our patients (and indeed all of life) are governed by the physics of nonequilibrium thermodynamics (i.e. systems of flow). Essential for our health continuously over time is our ability to constantly burn oxygen into carbon dioxide, which serves to continuously be dissipating a chemical energy gradient, and thus be continuously producing entropy. Illness is characterized by reduction in entropy production (both basal and maximal), and death by its cessation. Entropy production is defined physically by our heat production (due to both metabolism and consciousness) divided by our body temperature (i.e. $\mathrm{S}=\mathrm{Q} / \mathrm{T}$ ). Understanding the clinical importance of entropy production requires that we explore the thermodynamic physics of physiology.

In this article, these concepts are reviewed, discussed and hypotheses presented, believing they may offer novel and helpful insights regarding understanding and treatment of our patients, and merit further investigation. Nature's pursuit of optimal entropy production is hypothesized to lead to spontaneous ordered structures that define health, where physical and evolutionary forces together shape human entropy production. The origins and health of physiologic fractal structures as well as systemic properties such as healing and consciousness are discussed along with therapeutic implications. Experiments required to disprove these hypotheses are suggested. The aim is to stimulate critical appraisal and improved understanding of the physics of physiology in order to better care for our patients.

\section{Introduction to Entropy}

A brief review of the scientific concept of entropy is essential to begin. Thermodynamic Laws inform us that energy is always conserved (First Law), yet its quality degrades (as energy quality connotes its ability to perform work), as nature will seek to spread out or eliminate energy gradients (Second Law).(1,2) This loss of energy's ability to perform work is measured as entropy. Change in entropy of a system is universally positive; that is, loss of energy's ability to do work is irreversible, and provides directionality to time, always moving forward. Thus, entropy is produced. Microscopically, entropy production is probabilistic: for example, a system will pursues states with greater accessible microstates (i.e. microscopic configurations) that 
reflect more spreading out of gradients, resulting in greater entropy overall. If energy gradients exist, entropy production spontaneously occurs in order to irreversibly break down the gradient, as "nature abhors a gradient".(3) If this were the end of the story, we would observe that nature would simply degrade into maximally dispersed energy gradients (i.e. an orderless soup). However in striking contrast, nature and life are characterized by the continuous creation of remarkable ordered complex systems that are far from thermodynamic equilibrium. In fact, it is precisely by continuously producing entropy (i.e. degrading energy gradients) that life spontaneously creates its internal order.

\section{Entropy production and life}

All of us are living systems that must continuously import free energy (i.e. $\mathrm{O}_{2}$, glucose, $\mathrm{H}_{2} \mathrm{O}$, food), continuously export waste (i.e. $\mathrm{CO}_{2}$, urine, stool), necessarily continuously producing entropy to our environment, until we don't, signifying the end of our life. As originally highlighted by Edwin Schrödinger (1887-1961) in 1944,(4) life derives “order from order" from generation to generation (i.e. he postulated the necessity of a genetic code), and "order from disorder" (i.e. creating internal order only if accompanied by a greater release of entropy or disorder to the environment). As any thermodynamic body, our entropy production is equal to heat production $(\mathrm{Q})$ divided by temperature $(\mathrm{T})$. Studying entropy production in multiple living systems, Ichiro Aoki has noted that all living creatures go through a process of initial rising entropy production, then stability, followed by a period of slow decline, then a period of deterioration to zero, synonymous with death. $(5,6)$ This graphical representation of sigmoidal shaped initial rise in entropy production, followed by a gently falling plateau period, followed by loss during aging and death is precisely similar to the observed pattern of $\mathrm{VO}_{2}$ max levels during growth, middle age, illness and dying.(7-9) Indeed given the association between entropy production and heat production and metabolism, it suggests the origins of organ and cellular metabolic function may be fundamentally related to entropy production.

\section{Maximum Entropy Production}

Derived from atmospheric and multidisciplinary science, increasing evidence suggests that complex non-equilibrium systems, characterized by continuous energy dissipation (and thus entropy production) will adopt stable states involving maximal entropy production (given system 
constraints) such that the energy dissipation and entropy production will not only occur spontaneously, it will do so in the most efficient way possible. This Maximum Entropy Production Principle (MEPP) suggests that energy flow will naturally find the path that optimally dissipates the energy gradient, bound by the system's constraints, and will even lead to a system developing internal ordered structures, provided those structures enhance the energy flow. Although the MEPP has been questioned due to an apparent lack of clearly defined conditions required,(10-15) its application is supported in all complex non-equilibrium systems which clearly display "emergence". $(16,17)$ MEPP has demonstrated effectiveness in the solution of a broad variety of problems, $(16,18-21)$ and yet here, our focus is improved understanding of our patients and their care. To that end, we return to physiology, metabolism and structures associated with health.

\section{Entropy production and the Origin of Metabolism and Complex Structures}

A complex non-equilibrium system will adopt an internal state of greater complexity (i.e. complex order) if the state is associated with greater energy flow, which enables greater energy gradient dissipation, and thus greater entropy production; that is, ordered structures form to enhance energy flows. Examples are replete in nature, from Bénard cells (spontaneous convective flow in liquid layers) to spontaneous formation of a whirlpool that enhances the flow down the drain of a bathtub, to tornados, hurricanes and more. This physical force selects paths

of system change to select states that enhance energy flow. Metabolism, the burning of oxygen to carbon dioxide is precisely the principal means aerobic cells and organisms produce entropy. Indeed, reduction of oxygen provides for "close to the largest possible transfer of energy for each electron transfer reaction", and this steep thermodynamic gradient of oxygen is believed critical to the development of multicellular complexity.(22) Furthermore, if a cooperative collective of multiple cells is capable of greater entropy production, then nature's physical drive to enhance entropy production may help drive the spontaneous leap from single to multi-cellular organisms. Analogously, the growth of cities has been enabled by the capacity to allow for greater energy flow into the city, and clearance of waste from it. Supporting this principle, Chiasson has observed a remarkable pattern of increasing energy flow normalized by system size (measure in ergs per sec per gram) universally in inanimate and then animate non-equilibrium systems since the origin of the universe; in fact, energy flow density so closely correlates with system 
complexity, that is represents a reasonable measure of complexity itself.(23) Thus, according to MEPP, the origin of the complexity of oxygen burning metabolism has arisen from the dynamics of living systems adjusting themselves to lead to maximum entropy production.(24)

In addition to metabolic function, physiologic structures form spontaneously driven by MEPP. For example, the ubiquitous presence of fractal structures (i.e. those which demonstrate multiscale self-similarity) are hypothesized to originate because they optimize entropy production. Everywhere in nature, including anatomy and physiology, fractal physical structures spontaneously form, demonstrating their defining bounded multi-scale self-similarity, that is similar characteristic patterns (e.g. branching, waves) over many scales of magnitude within the bounds of the system. Spatially, fractal structures (e.g. trees, mountains, coastlines, river deltas, lightning, tornados, whirlpools) appear ubiquitously demonstrating bounded, measurable, multiscale self-similarity. Anatomically, alterations in these structures are associated with systemic change, known as illness in patients (e.g. altered tracheobronchial tree structure in asthma, $(25$, 26) altered vasculature in stroke, $(27,28)$ and altered CNS fractal dimensions in brain pathology(29)). Temporally, nature is also replete with complex time-series, which display power-law dynamics, again with bounded multi-scale self-similarity. These characteristics are found with heart rate variability (HRV) and respiratory rate variability (RRV), and whose complexity characteristics are preserved in health, and reduced with illness, stress and ageing.(30-53) Applying MEPP to understand the origin of fractal heart and respiratory rate dynamics, we previously hypothesized that the fractal structure of HRV and RRV develops as a self-organizing emergent event, spontaneously occurring and continuously enabling the system to optimize its entropy production.(54) Thus, nature's drive to optimize entropy production is hypothesized to explain why fractal structures are ubiquitous and spontaneously self-organizing. For example, fractal geometry of coastlines serve as an attractor, with coastlines universally and naturally converging to a fractal shape, as irregular coastlines help dampen sea waves.(55)All of this applies to the origin of complex structures observed in physical (i.e. mountain ranges, coastlines), geological (i.e. Richter's Law) systems as well as biologic systems in space (e.g. fractal vascular trees) and time (e.g. HRV, RRV). However biological systems have also been driven by a separate and complementary force, namely evolution. 


\section{Impact of Evolution}

If the drive to dissipate energy gradients leads to complex dissipative structures that are a natural physical phenomenon ubiquitous in nature from whirlpools to hurricanes to trees to cities, then what impact does evolution separately have in the biological world, where selection based on survival and reproductive potential has played a central role over millennia? It would seem that these two forces, namely physical and evolutionary, would lead to independent yet related impact. It is hypothesized that while nature's physical efforts serve to augment entropy production, complexity and function, evolution selects for an additional feature, namely adaptability or ability to tolerate increased workload, measurable by the capacity to augment entropy production if and when required. Both function and adaptability, measured by basal and maximal entropy production, are thus hypothesized to be a useful means to measure health. Basal entropy production is necessary for function, maintenance and repair (i.e. healing), whereas capacity to augment entropy production and augment work output would be required for adaptability, capacity to augment workload, to evade or respond to threats, either physical or illness related. Illness is hypothesized to be characterized by reduction in either resting and/or maximal oxygen consumption; however, if the reduction in maximal consumption is profound, then a compensatory elevation in resting energy expenditure may appear as a response, for example with $\operatorname{COPD}(56)$ or sepsis(57). Human entropy production is most readily estimated by oxygen consumption, reflecting metabolism and heat production, assuming stable temperature. Thus, overall health, reflecting both function and adaptability, is hypothesized to be related to either the ratio or difference between maximal oxygen consumption and resting energy expenditure. Supporting both resting and maximal entropy production ensures both function and adaptability are optimized, both important to overall health. While this exploration on nonequilibrium thermodynamics may seem already too complex, it does not end with metabolic thermodynamic entropy production; informational entropy production must also be considered.

\section{Informational Entropy Production}

Greater than any other living creature, we have developed a remarkably complex central nervous system that performs an additional and vital form of entropy production. The study of entropy has long since been performed in both information science and thermodynamics.(58) Formally 
linking the two, Landauer's principle states that any loss of information must be accompanied by entropy production in the environment,(59) now confirmed experimentally.(60-63) As the most cognitively advanced living creature, it is hypothesized that humans do not only produce entropy thermodynamically metabolically but also through information loss through our consciousness. To clarify, "loss of information" through consciousness connotes both a synthesis of data into meaning and storage with memory. Akin to diminishing the resolution of a photograph without losing its meaning, information loss is synonymous with entropy production, and is irreversible; and no process can result in a net gain of information over time.(64) When one translates an array of information or data into a shorter description indicating meaning or understanding, that synthesis of information connotes some loss of information, as the meanings or symbols we use to simplify the world never truly reflect all the detail of the real image.

As a thought experiment relevant to all of us, let us imagine the origins of consciousness in an unborn child approaching and then experiencing birth. Initial sensory information would be vast and largely uncorrelated, yet through repeated experiences, recurring patterns are detected, and meaning ascribed, as consciousness grows. Just considering visible sensory information, imagine the information initially exposed to a newborn infant's eye and its evolution over time; initially incomprehensible and uncorrelated, this vast array of visual sensory information is slowly reduced into meaning and memory as consciousness forms, remarkably and spontaneously in every single child. In a spontaneous emergent phenomenon, information is integrated and understood as shapes and symbols (e.g. faces, words) that effectively synthesize and reduce information into manageable quantities. The loss of uncorrelated information and gain of memories, classifications, theories, names, etc is hypothesized to represent human informational entropy production; thus, the emergence and the growth of consciousness is thus again a byproduct of nature's drive for maximal entropy production. While one imagines consciousness as a driver of creating information, its origins are derived from nature's aim to synthesize information and in so doing produce entropy. To summarize, dependent upon yet distinct from metabolism, the spontaneous emergence of human consciousness requires the processing, synthesis and storage of information, reflecting the ubiquitous phenomenon of nature's pursuit of entropy production. 


\section{Evaluation and Implications}

The hypotheses that fractal structures form to augment entropy production, that health is characterized by elevated baseline and maximal entropy production, and that human entropy production includes informational processing all merit rigorous evaluation. The association between complex fractal structures and flow may be evaluated by evaluating both in broadly disparate systems, analysing the growth in flow as well as complexity of physical model of energy flow (e.g. whirlpools, hurricanes), as well as explored with computational models. The hypothesis that health is characterized by both elevated baseline and capacity to augment entropy production may be tested by measuring resting and maximal heat production of a wide variety of humans. In addition, of greatest interest to clinicians, these hypotheses may be evaluated by their potential to improve patient care.

\section{Therapeutic Implications}

The clinically relevant hypothesis to evaluate is that targeted focus on optimizing our patient's entropy production, both at rest and maximally, will improve their health and clinical outcomes. As entropy production is measurable as heat production divided by temperature, and assuming temperature remains relatively constant, and heat production varies greatly with physical activity (which drives metabolism), entropy production over time is thus closely linked to metabolism over time. Heat production reaches maximal values at near peak exercise (i.e. $\mathrm{VO}_{2} \mathrm{max}$ ) maintainable for very short intervals; yet over longer periods, heat production is predominantly secondary to resting energy expenditure (REE).(65) Pursuing a strategy of optimizing resting and capacity for maximal entropy production is concordant with existing data and care. Athletes have both elevated resting energy expenditure, and the highest levels of maximal oxygen consumption, which can be enhanced with interval exercise training.(66) If oxygen consumption (and entropy production) is markedly reduced, efforts to augment it should be instituted, by restoring what limiting factor exists (e.g. limitation in cardiac output, oxygen content, perfusion or cellular consumption). In patients, ensuring adequate oxygen delivery and ventilation, renal, hepatic and intestinal waste removal, and early ambulation after critical illness and surgery, all represent foundational therapies that support metabolism and entropy production. If entropy production is required for our patients to generate internal order (and generating internal order 
might also be described as healing), then the body's ability to heal itself, in addition to the emergent appearance of fractal order occurring during embryogenesis, may simply reflect nature's physical drive for maximizing entropy production.

Given entropy production is heat production divided by temperature, therapeutic alteration in temperature offers an avenue to test these hypotheses, and to better understand physiologic temperature alteration. Hyperthermia or fever may be beneficial if it enhances entropy production or the potential for recovery (i.e. pathways leading back to recovery). Fever might thus be viewed as a means to overcome a potential barrier in order to stimulate the creation of such pathways, otherwise unavailable. As fever enhances metabolic rate and oxygen consumption $(60,67-69)$ as well as immune function, $(70,71)$ fever is hypothesized to be useful if it augments metabolism and heat production to a greater proportional extent than the rise in temperature, such that entropy production is increased; thus suppressing fever in routine infection may be harmful.(72, 73) However, in critically ill patients with markedly elevated basal entropy production (i.e. elevated REE), fever is unlikely to further augment metabolism, and thus may not be beneficial, even harmful.(74) If temperature increases without a greater proportional rise in heat production, overall entropy production is reduced. Analogously, cooling patients may help maintain or enhance entropy production, delivered locally or systemically, if the cooling does not further reduce metabolism and heat production. Certainly, hypothermia is beneficial when energy supplies are deficient, such as after cardiac arrest,(75) or for cryopreservation of tissues and embryos, when one wishes to decrease metabolism. In addition, cooling is helpful to avoid local temperature elevation after joint arthroplasty.(76) Thus, monitoring entropy production may offer a means to guide when hyperthermia or cooling are helpful and when they are not. In summary, temperature alteration is hypothesized to be therapeutic if it augments resting and maximal entropy production.

Given the association of fractal structures to optimal entropy production, then monitoring multiscale self-similar fractal structures may assist with monitoring of systemic properties helpful to forewarn and/or guide decision making, and second, restoring fractal structures pose a means to augment basal and maximal entropy production. Health is associated with fractal fluctuations of heart and respiratory rate, and illness and aging are associated with loss of fractal variation.(7780) Loss of fractal anatomy (e.g. emphysema, atherosclerosis) impairs internal organ specific 
dissipation of energy gradients. Continuous monitoring of scale-invariant variation to detect when it is altered may offer a means of detecting the early onset of illness, or help to guide decision-making. $(45,50,81,82)$ In addition, restoring scale-invariant life-support offers value in improving oxygen delivery; for example, improvement in jugular venous oxygen saturation is observed during rewarming from bypass with biologically variable pulsatile (vs. apulsatile or conventional pulsatile) cardiopulmonary bypass, $(83,84)$ and biologically variable ventilation improves arterial oxygenation $(85,86)$. Thus, monitoring and/or restoring fractal physiology to help make clinical decisions, and/or to directly enhance entropy production may both prove therapeutic.

Last, the therapeutic implications of restoring or enhancing information processing and storage as a vital form of informational entropy production merits brief discussion. Clearly, restoring basal entropy production (i.e. basal consciousness) is a key component to critical care medicine. Avoiding or using light sedation in critically ill patients is part of standard care,(87-89) as deep sedation is harmful. A focus on restoring functional consciousness would complement existing guidelines. The question of the potential benefit of enhancing both basal and maximal information processing is worthy of further exploration; are brief periods of intense efforts to utilize information processing and memory therapeutic? The impact of this approach in clinical psychology is beyond the scope of this initial discussion.

\section{Conclusions}

All living systems produce entropy to survive; after a period of growth in entropy production, we lose it slowly during aging or abruptly during illness, and its cessation signifies the end of life. Helping our patients to optimize their entropy production at rest and maximally may assist with health and healing. Physiological and clinical research is required to critically appraise these hypotheses, with the hope that new understanding leads to improved patient care.

\section{$\underline{\text { Declarations }}$}

\section{Ethics Approval and consent to participate}

Not applicable. 


\section{Consent for Publication}

Not applicable.

\section{Availability of Data and Materials}

Not applicable.

\section{Competing Interests}

Andrew JE Seely is the Founder and Chief Science Officer for Therapeutic Monitoring Systems, a company created to help commercialize waveform-based variability-derived clinical decision support software tools at the bedside, in order to improve care. He holds several patents related to utilizing variability monitoring to improve care.

\section{Funding Source}

This research did not receive any specific grant from funding agencies in the public, commercial, or not-for-profit sectors.

\section{Author's Contributions}

Dr. Andrew Seely is the sole author of this paper.

\section{Acknowledgements}

Special thanks to those who provided valuable feedback to the paper, including Kimberley Newman, Nicolas Brodeur, Christophe Herry, Nathan Scales, and Tim Ramsay, as well as those who contributed enormously to these ideas in the past, namely Peter Macklem and John Seely. Special thanks to remarkable collaborators at the University of Ottawa including Andre Longtin and Glen Kenny, and my peer mentor colleagues in thoracic surgery and critical care medicine. 


\section{References}

1. $\quad$ Atkins P. The Second Law: Scientific American Library; 1984. 230 p.

2. Lambert FL. Disorder--A Cracked Crutch for Supporting Entropy Discussions Journal of Chemistry Education. 2002;79:187.

3. Schneider ED, Sagan D. Into the Cool: Energy flow, Thermodynamics and Life. Chicago: University of Chicago Press books; 2005. 378 p.

4. Schrödinger E. What is life? Cambridge: Cambridge University Press 1944.

5. Aoki I. Entropy production in human life span: A thermodynamical measure for aging. Age. 1994;17(1):29-31.

6. Aoki I. Entropy Principle for the Development of Complex Biotic Systems. 1st edition, Organisms, Ecosystems, the Earth ed: Elsevier; 2012. 122 p.

7. Brown SJ, Ryan HJ, Brown JA. Age-Associated Changes In VO2 and Power Output - A Cross-Sectional Study of Endurance Trained New Zealand Cyclists. J Sports Sci Med. 2007;6(4):477-83.

8. Hawkins SA, Wiswell RA. Rate and Mechanism of Maximal Oxygen Consumption Decline with Aging: Implications for Exercise Training. Sports Med. 2003;33(12):877-88.

9. Plowman S, Smith D. Cardiovascular_Responses to Exercise Exercise Physiology for Health, Fitness, and Performance. 2nd ed. Baltimor MD: Lippincott Williams \& Wilkins; 2008.

10. Ross J, Corlan AD, Muller SC. Proposed principles of maximum local entropy production. J Phys Chem B. 2012;116(27):7858-65.

11. Vellela M, Qian H. Stochastic dynamics and non-equilibrium thermodynamics of a bistable chemical system: the Schlogl model revisited. J R Soc Interface. 2009;6(39):925-40.

12. Nicolis C. Stochastic resonance in multistable systems: the role of intermediate states. Phys Rev E Stat Nonlin Soft Matter Phys. 2010;82(1 Pt 1):011139.

13. Meysman FJ, Bruers S. Ecosystem functioning and maximum entropy production: a quantitative test of hypotheses. Philos Trans R Soc Lond B Biol Sci. 2010;365(1545):1405-16.

14. Polettini M. Fact-Checking Ziegler's Maximum Entropy Production Principle beyond the Linear Regime and towards Steady States. Entropy. 2013;15:2570-84.

15. Andresen B, Zimmermann EC, Ross J. Objections to a proposal on the rate of entropy production in systems far from equilibrium. J Chem Phys. 1984;81: 4676-7.

16. Martyushev LM. Entropy and Entropy Production: Old Misconceptions and New Breakthroughs. Entropy. 2013;15(1):1152-70.

17. Martyushev LM, Seleznev VD. The restrictions of the maximum entropy production principle. Physica A: Statistical Mechanics and its Applications. 2014;4410:17-21.

18. Kleidon A, Lorenz RD. Entropy production by earth system processes. In: Kleidon A, Lorenz RD, editors. In Non-Equilibrium Thermodynamics and The Production of Entropy: Life, Earth, and Beyond. Berlin, Germany: Springer Verlag; 2005.

19. Martyushev LM, Seleznev VD. Maximum entropy production principle in physics, chemistry and biology. Phys Rep. 2006;426:1-45.

20. Ozawa H, Ohmura A, Lorenz RD, Pujol T. The second law of thermodynamics and the global climate system: A review of the maximum entropy production principle. Rev Geophys 2003;41:1018-42.

21. Paltridge GW. Climate and thermodynamic systems of maximum dissipation. Nature. 1979;279:630-1. 
22. Koch LG, Britton SL. Aerobic metabolism underlies complexity and capacity. J Physiol. 2008;586(1):83-95.

23. Chiasson EJ. Cosmic Evolution: The Rise of Complexity in Nature. Cambridge: Harvard University Press; 2001. 274 p.

24. Niven RK. Steady state of a dissipative flow-controlled system and the maximum entropy production principle. Phys Rev E Stat Nonlin Soft Matter Phys. 2009;80(2 Pt 1):021113.

25. Boser SR, Park H, Perry SF, Menache MG, Green FH. Fractal geometry of airway remodeling in human asthma. American journal of respiratory and critical care medicine. 2005;172(7):817-23.

26. Gupta S, Hartley R, Khan UT, Singapuri A, Hargadon B, Monteiro W, et al. Quantitative computed tomography-derived clusters: redefining airway remodeling in asthmatic patients. J Allergy Clin Immunol. 2014;133(3):729-38 e18.

27. Doubal FN, MacGillivray TJ, Patton N, Dhillon B, Dennis MS, Wardlaw JM. Fractal analysis of retinal vessels suggests that a distinct vasculopathy causes lacunar stroke. Neurology. 2010;74(14):1102-7.

28. Cavallari M, Falco T, Frontali M, Romano S, Bagnato F, Orzi F. Fractal analysis reveals reduced complexity of retinal vessels in CADASIL. PLoS One. 2011;6(4):e19150.

29. Seely A, Newman KD, Herry C. Fractal Structure and Entropy Production within the Central Nervous System. Entropy. 2014;16(8): 4497-520.

30. Seely AJ, Macklem PT. Complex systems and the technology of variability analysis. Critical care (London, England). 2004;8(6):R367-R84-R-R84.

31. Axelrod S, Lishner M, Oz O, Bernheim J, Ravid M. Spectral analysis of fluctuations in heart rate: an objective evaluation of autonomic nervous control in chronic renal failure. Nephron. 1987;45(3):202-6.

32. Bonaduce D, Petretta M, Marciano F, Vicario ML, Apicella C, Rao MA, et al. Independent and incremental prognostic value of heart rate variability in patients with chronic heart failure. Am Heart J. 1999;138(2 Pt 1):273-84.

33. Guzzetti S, Mezzetti S, Magatelli R, Porta A, De Angelis G, Rovelli G, et al. Linear and non-linear $24 \mathrm{~h}$ heart rate variability in chronic heart failure. Auton Neurosci. 2000;86(1-2):1149.

34. Huang J, Sopher SM, Leatham E, Redwood S, Camm AJ, Kaski JC. Heart rate variability depression in patients with unstable angina. Am Heart J. 1995;130(4):772-9.

35. Lishner M, Akselrod S, Avi VM, Oz O, Divon M, Ravid M. Spectral analysis of heart rate fluctuations. A non-invasive, sensitive method for the early diagnosis of autonomic neuropathy in diabetes mellitus. Journal of the autonomic nervous system. 1987;19(2):119-25. 36. Mussalo H, Vanninen E, Ikaheimo R, Laitinen T, Laakso M, Lansimies E, et al. Heart rate variability and its determinants in patients with severe or mild essential hypertension. Clin Physiol. 2001;21(5):594-604.

37. Poulsen SH, Jensen SE, Moller JE, Egstrup K. Prognostic value of left ventricular diastolic function and association with heart rate variability after a first acute myocardial infarction. Heart (British Cardiac Society). 2001;86(4):376-80.

38. van Boven AJ, Jukema JW, Haaksma J, Zwinderman AH, Crijns HJ, Lie KI. Depressed heart rate variability is associated with events in patients with stable coronary artery disease and preserved left ventricular function. REGRESS Study Group. Am Heart J. 1998;135(4):571-6. 39. van de Borne P, Montano N, Pagani M, Oren R, Somers VK. Absence of low-frequency variability of sympathetic nerve activity in severe heart failure. Circulation. 1997;95(6):1449-54. 
40. Costa M, Goldberger AL, Peng CK. Multiscale entropy analysis of complex physiologic time series. Phys Rev Lett. 2002;89(6):068102.

41. Goldberger AL, Amaral LA, Glass L, Hausdorff JM, Ivanov PC, Mark RG, et al. PhysioBank, PhysioToolkit, and PhysioNet: components of a new research resource for complex physiologic signals. Circulation. 2000;101(23):E215-20.

42. Bravi A, Longtin A, Seely AJ. Review and classification of variability analysis techniques with clinical applications. Biomed Eng Online. 2011;10:90.

43. Odemuyiwa O, Malik M, Farrell T, Bashir Y, Poloniecki J, Camm J. Comparison of the predictive characteristics of heart rate variability index and left ventricular ejection fraction for all-cause mortality, arrhythmic events and sudden death after acute myocardial infarction. The American journal of cardiology. 1991;68(5):434-9.

44. Rello J, Ollendorf DA, Oster G, Vera-Llonch M, Bellm L, Redman R, et al. Epidemiology and outcomes of ventilator-associated pneumonia in a large US database.[see comment]. Chest. 2002;122(6):2115-21.

45. Ahmad S, Ramsay T, Huebsch L, Flanagan S, McDiarmid S, Batkin I, et al. Continuous multi-parameter heart rate variability analysis heralds onset of sepsis in adults. PLoS ONE. 2009;4(8):e6642.

46. Buchan CA, Bravi A, Seely AJ. Variability Analysis and the Diagnosis, Management, and Treatment of Sepsis. Curr Infect Dis Rep. 2012.

47. Bravi A, Green G, Longtin A, Seely AJ. Monitoring and identification of sepsis development through a composite measure of heart rate variability. PLoS One. 2012;7(9):e45666.

48. Green GC, Bradley B, Bravi A, Seely AJ. Continuous multiorgan variability analysis to track severity of organ failure in critically ill patients. J Crit Care. 2013;28(5):879 e1-11.

49. Brack T, Jubran A, Tobin MJ. Dyspnea and decreased variability of breathing in patients with restrictive lung disease. American journal of respiratory and critical care medicine. 2002;165(9):1260-4.

50. Seely AJ, Bravi A, Herry C, Green G, Longtin A, Ramsay T, et al. Do heart and respiratory rate variability improve prediction of extubation outcomes in critically ill patients? Critical care (London, England). 2014;18(2):R65.

51. Papaioannou VE, Chouvarda I, Maglaveras N, Dragoumanis C, Pneumatikos I. Changes of heart and respiratory rate dynamics during weaning from mechanical ventilation: a study of physiologic complexity in surgical critically ill patients. J Crit Care. 2011;26(3):262-72.

52. Savi A, Teixeira C, Silva JM, Borges LG, Pereira PA, Pinto KB, et al. Weaning predictors do not predict extubation failure in simple-to-wean patients. J Crit Care. 2012;27(2):221 e1-8.

53. Papaioannou VE, Chouvarda IG, Maglaveras NK, Pneumatikos IA. Study of multiparameter respiratory pattern complexity in surgical critically ill patients during weaning trials. BMC Physiol. 2011;11:2.

54. Seely AJ, Macklem P. Fractal variability: an emergent property of complex dissipative systems. Chaos (Woodbury, NY. 2012;22(1):013108.

55. Sapoval B, Baldassarri A, Gabrielli A. Self-stabilized fractality of seacoasts through damped erosion. Phys Rev Lett. 2004;93(9):098501.

56. Schols AM, Fredrix EW, Soeters PB, Westerterp KR, Wouters EF. Resting energy expenditure in patients with chronic obstructive pulmonary disease. Am J Clin Nutr. 1991;54(6):983-7. 
57. Frankenfield DC, Wiles CE, 3rd, Bagley S, Siegel JH. Relationships between resting and total energy expenditure in injured and septic patients. Crit Care Med. 1994;22(11):1796-804.

58. Parrondo JMR, Horowitz JM, Sagawa T. Thermodynamics of information. Nature Physics. 2015;11:131-9.

59. Landauer R. Irreversibility and Heat Generation in the Computing Process. IBM Journal of Research and Development. 1961;5(3):183-91.

60. Standardization of spirometry-1987 update:statement of the American Thoracic Society. Am Rev Respir Dis 1987;136:1285-98.

61. Berut A, Arakelyan A, Petrosyan A, Ciliberto S, Dillenschneider R, Lutz E. Experimental verification of Landauer's principle linking information and thermodynamics. Nature. 2012;483(7388):187-9.

62. Jun Y, Gavrilov M, Bechhoefer J. High-precision test of Landauer's principle in a feedback trap. Phys Rev Lett. 2014;113(19):190601.

63. Hong J, Lambson B, Dhuey S, Bokor J. Experimental test of Landauer's principle in single-bit operations on nanomagnetic memory bits. Sci Adv. 2016;2(3):e1501492.

64. Duncan TL, Semura JS. Information Loss as a Foundational Principle for the Second Law of Thermodynamics. Foundations of Physics 2007;37(12):1767-73.

65. Aoki I. Entropy production in living systems: from organisms to ecosystems. Thermochimica Acta. 1995;250(2):359-70.

66. Westerterp KR. Control of energy expenditure in humans. Eur J Clin Nutr. 2017;71(3):340-4.

67. Frankenfield DC, Smith JS, Jr., Cooney RN, Blosser SA, Sarson GY. Relative association of fever and injury with hypermetabolism in critically ill patients. Injury. 1997;28(910):617-21.

68. Manthous CA, Hall JB, Olson D, Singh M, Chatila W, Pohlman A, et al. Effect of cooling on oxygen consumption in febrile critically ill patients. American journal of respiratory and critical care medicine. 1995;151(1):10-4.

69. Haupt MT, Rackow EC. Adverse effects of febrile state on cardiac performance. Am Heart J. 1983;105(5):763-8.

70. Nahas GG, Tannieres ML, Lennon JF. Direct measurement of leukocyte motility: effects of $\mathrm{pH}$ and temperature. Proc Soc Exp Biol Med. 1971;138(1):350-2.

71. Dinarello CA, Bernheim HA, Duff GW, Le HV, Nagabhushan TL, Hamilton NC, et al. Mechanisms of fever induced by recombinant human interferon. J Clin Invest. 1984;74(3):90613.

72. Sugimura T, Fujimoto T, Motoyama H, Maruoka T, Korematu S, Asakuno Y, et al. Risks of antipyretics in young children with fever due to infectious disease. Acta Paediatr Jpn. 1994;36(4):375-8.

73. Graham NM, Burrell CJ, Douglas RM, Debelle P, Davies L. Adverse effects of aspirin, acetaminophen, and ibuprofen on immune function, viral shedding, and clinical status in rhinovirus-infected volunteers. J Infect Dis. 1990;162(6):1277-82.

74. Drewry AM, Ablordeppey EA, Murray ET, Stoll CRT, Izadi SR, Dalton CM, et al. Antipyretic Therapy in Critically Ill Septic Patients: A Systematic Review and Meta-Analysis. Crit Care Med. 2017;45(5):806-13.

75. Arrich J, Holzer M, Havel C, Müllner M, Herkner H. Hypothermia for neuroprotection in adults after cardiopulmonary resuscitation. Cochrane Database of Systematic Reviews 2016(2). 
76. Adie S, Kwan A, Naylor JM, Harris IA, Mittal R. Cryotherapy following total knee replacement.. Cochrane Database of Systematic Reviews. 2021(9).

77. Goldberger AL. Fractal variability versus pathologic periodicity: complexity loss and stereotypy in disease. Perspect Biol Med. 1997;40(4):543-61.

78. Goldberger AL. Nonlinear Dynamics, Fractals, and Chaos Theory: Implications for Neuroautonomic Heart Rate Control in Health and Disease. Bolis CL LJ, eds., editor. Geneva: World Health Organization; 1999.

79. Goldberger AL, Peng CK, Lipsitz LA. What is physiologic complexity and how does it change with aging and disease? Neurobiol Aging. 2002;23(1):23-6.

80. West BJ. Physiology in fractal dimensions: error tolerance. Ann Biomed Eng. 1990;18(2):135-49.

81. Barnaby DP, Fernando SM, Herry CL, Scales NB, Gallagher EJ, Seely AJE. Heart Rate Variability, Clinical and Laboratory Measures to Predict Future Deterioration in Patients Presenting with Sepsis. Shock. 2018.

82. Samsudin MI, Liu N, Prabhakar SM, Chong SL, Kit Lye W, Koh ZX, et al. A novel heart rate variability based risk prediction model for septic patients presenting to the emergency department. Medicine (Baltimore). 2018;97(23):e10866.

83. Mutch WA, Lefevre GR, Thiessen DB, Girling LG, Warrian RK. Computer-controlled cardiopulmonary bypass increases jugular venous oxygen saturation during rewarming. The Annals of thoracic surgery. 1998;65(1):59-65.

84. Mutch WA, Warrian RK, Eschun GM, Girling LG, Doiron L, Cheang MS, et al. Biologically variable pulsation improves jugular venous oxygen saturation during rewarming. The Annals of thoracic surgery. 2000;69(2):491-7.

85. McMullen MC, Girling LG, Graham MR, Mutch WA. Biologically variable ventilation improves oxygenation and respiratory mechanics during one-lung ventilation. Anesthesiology. 2006;105(1):91-7.

86. Mutch WA, Buchman TG, Girling LG, Walker EK, McManus BM, Graham MR. Biologically variable ventilation improves gas exchange and respiratory mechanics in a model of severe bronchospasm. Crit Care Med. 2007;35(7):1749-55.

87. Barr J, Fraser GL, Puntillo K, Ely EW, Gelinas C, Dasta JF, et al. Clinical practice guidelines for the management of pain, agitation, and delirium in adult patients in the intensive care unit. Crit Care Med. 2013;41(1):263-306.

88. Mehta S, Burry L, Cook D, Fergusson D, Steinberg M, Granton J, et al. Daily sedation interruption in mechanically ventilated critically ill patients cared for with a sedation protocol: a randomized controlled trial. JAMA. 2012;308(19):1985-92.

89. Strom T, Martinussen T, Toft P. A protocol of no sedation for critically ill patients receiving mechanical ventilation: a randomised trial. Lancet. 2010;375(9713):475-80. 


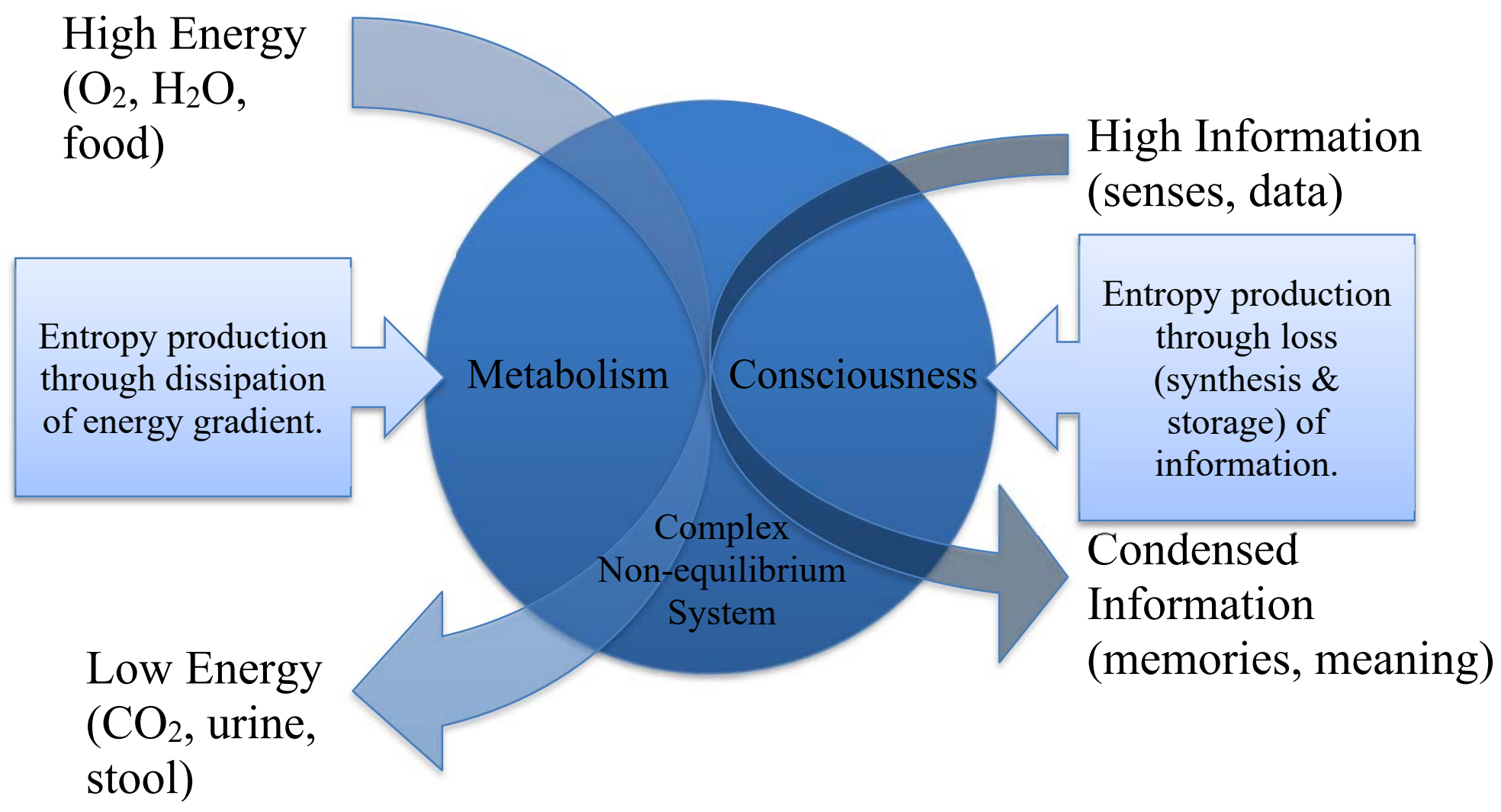

Figure 1: Complex adaptive dissipative cognitive system 
Table 1: Key concepts

\begin{tabular}{|c|c|}
\hline Entropy Production & $\begin{array}{l}\text { - Entropy is a measure of quality of energy (i.e. its ability to perform work). } \\
\text { - While energy is conserved, energy gradients are universally and irreversibly dispersed, producing entropy (i.e. } \\
\text { loss of energy quality). } \\
\text { - Complex non-equilibrium systems that are continuously breaking down energy gradients seek to augment their } \\
\text { entropy production (i.e. MEPP). } \\
\text { Entropy production equals heat production (Q) divided by temperature (T); however human heat production is } \\
\text { largely determined by metabolism, and is greatly impacted by temperature (e.g. fever drives increased } \\
\text { metabolism). } \\
\text { In all living structures, an initial growth in entropy production is observed, followed by a plateau, then a fall, } \\
\text { and its cessation occurs with death. }\end{array}$ \\
\hline Physical structures & $\begin{array}{l}\text { - Fractal structures in nature (i.e. bounded multiscale self-similarity) form spontaneously in order to optimize } \\
\text { entropy production. } \\
\text { - Fractal anatomic (i.e. tree-like) and temporal (e.g. heart rate variability) structures found in human physiology } \\
\text { are essential for optimal systemic entropy production. }\end{array}$ \\
\hline Impact of Evolution & $\begin{array}{l}\text { - The evolutionary drive for enhanced function and adaptability is hypothesized to select states with both robust } \\
\text { basal entropy production and the capacity to augment it when required. }\end{array}$ \\
\hline $\begin{array}{l}\text { Informational } \\
\text { Entropy Production }\end{array}$ & $\begin{array}{l}\text { - Humans also produce entropy through the synthesis and storage of information into meaning and memory } \\
\text { within the central nervous system. } \\
\text { - The origin of consciousness may reflect natures drive to produce entropy. }\end{array}$ \\
\hline Health & $\begin{array}{l}\text { - Overall human health, reflecting both function and adaptability, is hypothesized to be related to elevated both } \\
\text { resting and maximal entropy production, estimable by the basal resting energy expenditure and maximal } \\
\text { oxygen consumption. }\end{array}$ \\
\hline Illness & $\begin{array}{l}\text { - Breakdown of fractal structures in space (i.e. vascular networks, tracheobronchial tree) or time (i.e. heart rate } \\
\text { variability) occurs with illness. } \\
\text { - Illness and aging are associated with either a decrease in basal or maximal entropy production, or both. }\end{array}$ \\
\hline $\begin{array}{l}\text { Therapeutic } \\
\text { Implications }\end{array}$ & $\begin{array}{l}\text { - Optimizing our patient's entropy production, both at rest and maximally, will improve their health and clinical } \\
\text { outcomes, including both metabolism and consciousness. } \\
\text { - Monitoring loss of fractal variability to predict clinical outcomes may be useful to assist clinical decision- } \\
\text { making. } \\
\text { - Restoring fractal physiology through biologically variable life support may be useful to enhance entropy } \\
\text { production. } \\
\text { Therapeutic temperature alteration may be guided my monitoring the impact on heat production divided by } \\
\text { temperature; hyperthermia or cooling may be beneficial if they enhance entropy production. }\end{array}$ \\
\hline
\end{tabular}




\section{Figures}

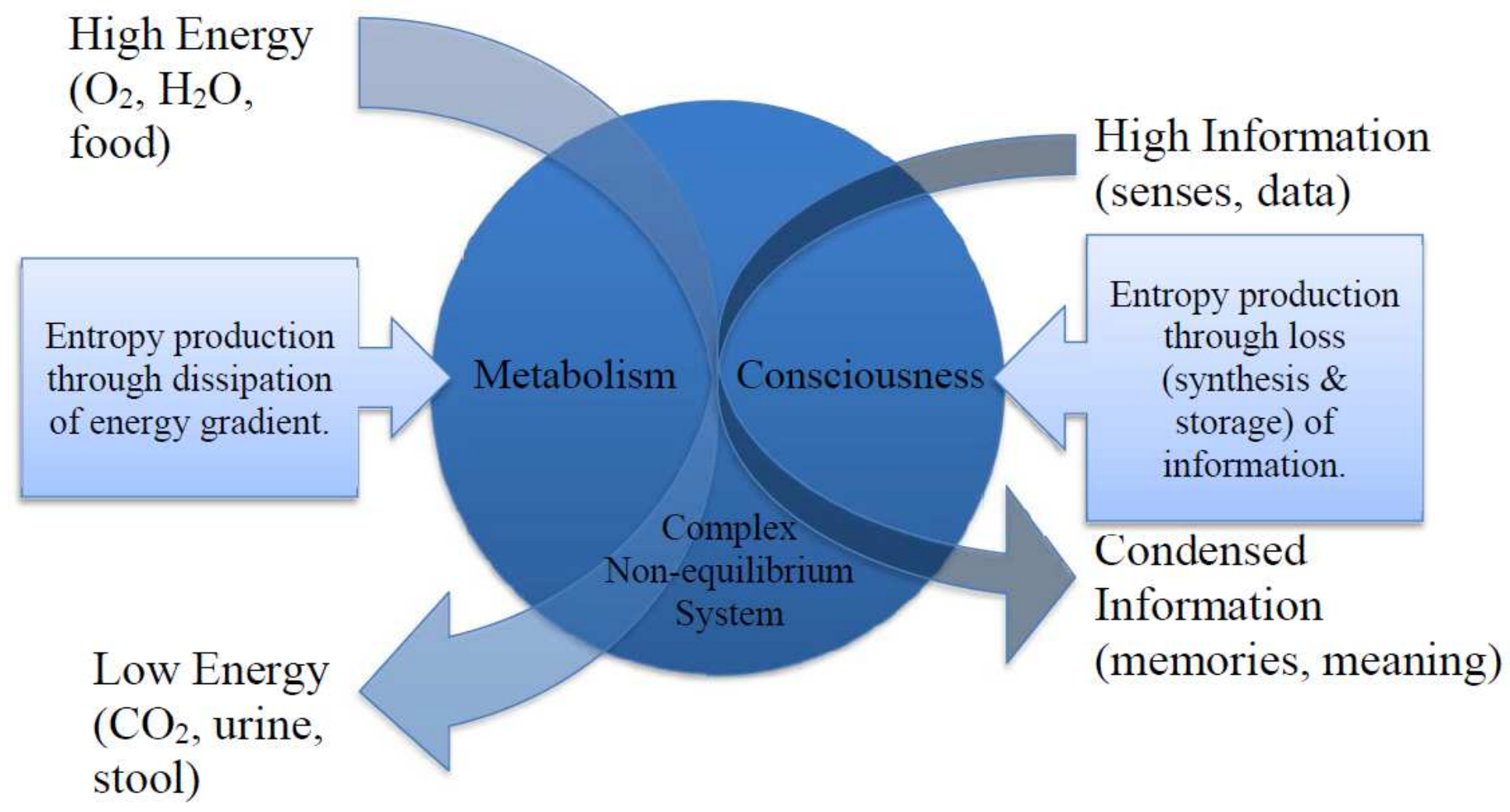

Figure 1

Complex adaptive dissipative cognitive system 